\title{
INEQUALITIES FOR SOME SPECIAL FUNCTIONS-II
}

\author{
S. K. BISSU AND C. M. JOSHI
}

\begin{abstract}
Some inequalities for Bessel functions, modified Bessel functions of the first kind and of their ratios involving both lower and upper bounds are given. The inequalities improve the results of earlier authours. Also incorporated in the discussion are some inequalities for the ratios of confluent hypergeometric functions of one variable.
\end{abstract}

\section{Introduction.}

For $J_{\nu}(x)$, the Bessel function of first kind, Paris [14] proved an inequality when the argument is less than the order. The inequality obtained by him is sharp for $x \rightarrow 1$. Similar results have been established by Laforgia [8] for general cylinder functions

$$
C_{\nu}(x, \alpha)=\cos \alpha J_{\nu}(x)-\sin \alpha Y_{\nu}(x), \quad 0 \leq \alpha<\pi,
$$

where $Y_{\nu}(x)$ is the Bessel functions of second kind. Recently Laforgia and Mathis [11] have proved the inequalities

$$
\begin{gathered}
\frac{J_{\nu}(x)}{J_{\nu}(y)}>\left(\frac{x}{y}\right)^{\nu^{2}+\frac{1}{4}} \exp \left\{\frac{y^{2}-x^{2}}{16}\right\}, \nu>0,0<x<y \leq \nu, \\
\frac{J_{\nu}(x)}{J_{\nu}(y)}<\left(\frac{x}{y}\right)^{\frac{3}{16}+\frac{3}{2} \nu^{2}-\nu^{4}} \exp \left\{\frac{\left(y^{2}-x^{2}\right)}{36}\left[7-4 \nu^{2}+\frac{1}{5}\left(y^{2}+x^{2}\right)\right]\right\}, \\
\text { for } 0<x<y<\gamma, \nu \geq \frac{1}{2},
\end{gathered}
$$

$\gamma=\min \left\{\sqrt{\left(\nu^{2}-\frac{1}{4}\right)}, t_{1}\right\}, t_{1}$ possibly the first positive zero of

$$
16 t^{2}+40\left(7-4 \nu^{2}\right) t^{2}+45\left(16 \nu^{4}-24 \nu^{2}-11\right)
$$

Received Jaunary 25, 1994; revised July 8, 1994.

1991 Mathematics Subject Classification. Primary 33C10, 33C15; Secondary 26 D99.

Key words and phrases. Bessel and modified Bessel functions, inequalities, confluent hypergeometric functions, recurrence relations. 
and similar results for $I_{\nu}(x)$ (see also Laforgia [10]).

Inequalities for the ratios of modified Bessel functions of the first and second kind are available in the literature. For instance, the inequality for the ratio $K_{\nu}(x) / I_{\nu}(x)$ have been used by Rosenthal [15] and Ross [16] in determining the stability of fluid motion. However, for modified Bessel functions of first kind $I_{\nu}(x)$, Bordelon [3] and Ross [17] independently proved the inequality

$$
\exp \{x-y\}\left(\frac{x}{y}\right)^{\nu}<\frac{I_{\nu}(x)}{I_{\nu}(y)}<\exp \{y-x\}\left(\frac{x}{y}\right)^{\nu}, \nu>0,0<x<y .
$$

This inequality has been improved upon by Paris [14] in the form

$$
\exp \{x-y\}\left(\frac{x}{y}\right)^{\nu}<\frac{I_{\nu}(x)}{I_{\nu}(y)}<\left(\frac{x}{y}\right)^{\nu}, \nu>-\frac{1}{2}, 0<x<y .
$$

In this paper we are concerned with some further inequalities for $J_{\nu}(x)$ and $I_{\nu}(x)$. In Section 2, we have obtained the lower and upper bounds for $J_{\nu}(x), \nu>-1, x>0$, which are sharper than those given in $[2,4,11,17,18$ and 19]. This inequality then leads to the inequalities for the ratios of $J_{\nu}(x) / J_{\nu}(y)$ and $J_{\nu+1}(x) / J_{\nu}(x)$. In Section 3 , inequalities for $I_{\nu}(x), I_{\nu}(x) / I_{\nu}(y)$ and $I_{\nu+1}(x) / I_{\nu}(x)$, have been obtained and sharpness of these results with the existing ones have been pointed out numerically. The paper is concluded by appending the bounds for the ratios ${ }_{1} F_{1}(a ; b ;-x) /{ }_{1} F_{1}(c ; d ;-y),{ }_{1} F_{1}(a ; b ; x) /{ }_{1} F_{1}(c ; d ; y)$ and ${ }_{1} F_{1}(a ; b ; x) /{ }_{1} F_{1}(a ; c ; x)$ and it is verified numerically that these bounds provide improvement over Bordelon's results ([3], p.668 (6)) in $0<x<1,0<y<1$.

\section{Inequalities for Bessel functions}

The function

$$
{ }_{0} F_{1}(-; \alpha ;-z)=\sum_{m=0}^{\infty} \frac{(-z)^{m}}{m !(\alpha)_{m}}, \quad \alpha>0
$$

satisfies the inequality

$$
1-\frac{z}{\alpha}<{ }_{0} F_{1}(-; \alpha ;-z)<1-\frac{z}{\alpha}+\frac{z^{2}}{2(\alpha)_{2}},
$$

which is valid for $0<z<3(\alpha+2)$ by a usual argument for a series of alternating signs with terms of magnitude tending to zero, by grouping the terms in pairs whose sum is positive.

Now (2.1) can be put in the form

$$
\begin{aligned}
1-\frac{x^{2}}{4(\nu+1)}< & \frac{\Gamma(\nu+1)}{(x / 2)^{\nu}} J_{\nu}(x)<1-\frac{x^{2}}{4(\nu+1)}+\frac{x^{4}}{32(\nu+1)(\nu+2)}, \\
& \nu>-1,0<x<2(3(\nu+3))^{1 / 2}
\end{aligned}
$$


which is the same as that of Joshi and Bissu [7] but the method of proof is different.

The inequality (2.2) is sharper than the inequality

$$
\frac{\Gamma(\nu+1)}{(x / 2)^{\nu}} J_{\nu}(x)<\exp \left\{x^{2} / 4(\nu+1)\right\}, \quad \nu \geq 0, x>0
$$

given in Watson [19]. Further, this inequality for $\nu=\alpha-\frac{1}{2}$ improves the inequality

$$
\begin{gathered}
J_{\alpha-1 / 2}(x)<(x / 2)^{\alpha-1 / 2} \Gamma(\alpha+1 / 2)^{-1}\left\{1+\frac{x^{2}}{\alpha(2 \alpha+1)}\right\}^{-\alpha / 2} \\
0.065 \leq \alpha<1, x>0
\end{gathered}
$$

of Common [4] and that of Askey [2]

$$
J_{\alpha}(x)<\left(\frac{x}{2}\right)^{\alpha} \Gamma(\alpha+1)^{-1}, \quad \alpha \geq-1 / 2, x>0
$$

when $0<x \leq 2(2(\nu+2))^{1 / 2}$.

Next, (2.2) can also be expressed as

$$
\begin{aligned}
\frac{1}{\left[1-\frac{y^{2}}{4(\nu+1)}+\frac{y^{4}}{32(\nu+1)(\nu+2)}\right]}<\frac{(y / 2)^{\nu}}{\Gamma(\nu+1) J_{\nu}(y)}<\frac{1}{\left[1-\frac{y^{2}}{4(\nu+1)}\right]} \\
\\
\nu>-1,0<y \leq 2 \sqrt{(\nu+1)} .
\end{aligned}
$$

Therefore, combining (2.2) and (2.6) we have

$$
\begin{aligned}
\left(\frac{x}{y}\right)^{\nu}\left[\frac{\left(1-\frac{x^{2}}{4(\nu+1)}\right)}{1-\frac{y^{2}}{4(\nu+1)}+\frac{y^{4}}{32(\nu+1)(\nu+2)}}\right] & <\frac{J_{\nu}(x)}{J_{\nu}(y)} \\
& <\left(\frac{x}{y}\right)^{\nu}\left[\frac{1-\frac{x^{2}}{4(\nu+1)}+\frac{x^{4}}{32(\nu+1)(\nu+2)}}{1-\frac{y^{2}}{4(\nu+1)}}\right], \\
\nu>-1, x>0,0<y \leq 2 \sqrt{(\nu+1)} &
\end{aligned}
$$

and similarly

$$
\begin{aligned}
& \frac{x}{2(\nu+1)}\left[\frac{\left(1-\frac{x^{2}}{4(\nu+2)}\right)}{\left.1-\frac{x^{2}}{4(\nu+1)}+\frac{x^{4}}{32(\nu+1)(\nu+2)}\right]}\right.<\frac{J_{\nu+1}(x)}{J_{\nu}(x)} \\
&<\frac{x}{2(\nu+1)}\left[\frac{1-\frac{x^{2}}{4(\nu+2)}+\frac{x^{4}}{32(\nu+2)(\nu+3)}}{1-\frac{x^{2}}{4(\nu+1)}}\right], \\
& \nu>-1,0<x \leq 2 \sqrt{(\nu+1)} .
\end{aligned}
$$


This inequality along with the recurrence relation

$$
\frac{\nu}{2}-\frac{J_{\nu}^{\prime}(z)}{J_{\nu}(z)}=\frac{\dot{J}_{\nu+1}(z)}{J_{\nu}(z)}
$$

and integration over $(x, y)$ then leads to the inequality

$$
\begin{gathered}
\frac{J_{\nu}(x)}{J_{\nu}(y)}<\left(\frac{x}{y}\right)^{\nu} \exp \left\{\frac{(\nu+5)\left(y^{2}-x^{2}\right)}{8(\nu+2)(\nu+3)}+\frac{x^{4}-y^{4}}{16(\nu+2)(\nu+3)}\right\}\left[\frac{4(\nu+1)-x^{2}}{4(\nu+1)-y^{2}}\right]^{\frac{2(\nu+3)+(\nu+1)^{2}}{2(\nu+2)(\nu+3)}} \\
\nu>-1,0<x<y<2(\nu+1)^{1 / 2}
\end{gathered}
$$

Here it is observed that (2.7) and (2.9) give improved bounds and hold in the extended domain than that of Laforgia and Mathis [11] and of Joshi and Bissu [6].

\section{Inequalities for Modified Bessel Functions}

Proceeding in a similar manner as that of (2.2), we have

$$
\begin{aligned}
\left(1+\frac{x^{2}}{4(\nu+1)}\right)<{ }_{0} F_{1}\left(-; \nu+1 ; x^{2} / 4\right) & <1+\frac{x^{2} / 4}{\nu+1}\left(1+\frac{x^{2} / 4}{2}+\frac{x^{4} / 4^{2}}{2^{2}}+\cdots\right) \\
& <1+\frac{x^{2} / 4}{\nu+1}\left(1+\frac{1}{2}+\frac{1}{2^{2}}+\cdots\right) \\
& =1+\frac{x^{2}}{2(\nu+1)}, \quad 0<x<2 .
\end{aligned}
$$

Hence, we have

$$
\begin{gathered}
\left(1+\frac{x^{2}}{4(\nu+1)}\right)<\frac{\Gamma(\nu+1)}{(x / 2)^{\nu}} \dot{I}_{\nu}(x)<\left(1+\frac{x^{2}}{2(\nu+1)}\right), \\
\nu>-1,0<x<2
\end{gathered}
$$

which holds in the extended domain $1 \leq x<2$ than that of Joshi and Bissu [7] and improves the lower and upper bounds of Luke's [12] inequality

$$
1<\frac{\Gamma(\nu+1)}{(x / 2)^{\nu}} I_{\nu}(x)<\left(e^{-x}+e^{x}\right) / 2, \nu>-1 / 2, x>0
$$

and is quite sharp when $x \rightarrow 0$.

Proceeding as before, (3.1) admits the forms

$$
\begin{aligned}
\frac{(x / y)^{\nu}\left(1+\frac{x^{2}}{4(\nu+1)}\right)}{\left(1+\frac{y^{2}}{2(\nu+1)}\right)}<\frac{I_{\nu}(x)}{I_{\nu}(y)}<\frac{(x / y)^{\nu}\left(1+\frac{x^{2}}{2(\nu+1)}\right)}{\left(1+\frac{y^{2}}{4(\nu+1)}\right)} \\
\\
\nu>-1,0<x<2,0<y<2
\end{aligned}
$$


and

$$
\begin{gathered}
\frac{x\left(1+\frac{x^{2}}{4(\nu+2)}\right)}{2(\nu+1)\left(1+\frac{x^{2}}{2(\nu+1)}\right)}<\frac{I_{\nu+1}(x)}{I_{\nu}(x)}<\frac{x\left(1+\frac{x^{2}}{2(\nu+2)}\right)}{2(\nu+1)\left(1+\frac{x^{2}}{4(\nu+1)}\right)} \\
\nu>-1,0<x<2 .
\end{gathered}
$$

The inequality (3.4), the reccurence relation [19, p.79]

$$
\frac{I_{\nu}^{\prime}(z)}{I_{\nu}(z)}-\frac{\nu}{z}=\frac{I_{\nu+1}(z)}{I_{\nu}(z)}
$$

and integration over $(x, y)$ give the inequality

$$
\begin{aligned}
& \quad\left(\frac{x}{y}\right)^{\nu} \exp \left\{\frac{x^{2}-y^{2}}{2(\nu+2)}\right\}\left[\frac{4(\nu+1)+y^{2}}{4(\nu+1)+x^{2}}\right]^{\frac{\nu}{(\nu+2)}} \\
& <\frac{I_{\nu}(x)}{I_{\nu}(y)} \\
& <\left(\frac{x}{y}\right)^{\nu} \exp \left\{\frac{x^{2}-y^{2}}{8(\nu+2)}\right\}\left[\frac{2(\nu+1)+x^{2}}{2(\nu+1)+y^{2}}\right]^{\frac{\nu+3}{4(\nu+2)}}, \\
& \quad \nu>-1,0<x<y<2 .
\end{aligned}
$$

Numerically, for the set of values $x=.4, y=.8, \nu=1,(3.6)$ gives

$$
0.4704361<\frac{I_{\nu}(x)}{I_{\nu}(y)}=0.4713405<0.4759643
$$

whereas Paris's ([14], p.204(4)) inequality gives

$$
0.33516<\frac{I_{\nu}(x)}{I_{\nu}(y)}=0.471305<0.5
$$

and that of Laforgia ([9], p.264 and [10] p.386)

$$
0.33516<\frac{I_{\nu}(x)}{I_{\nu}(y)}=0.471305<1.34064
$$

The results obtained above can be extended when $x \geq 2$. For this let us consider the modified. Bessel function of the first kind

$$
I_{\nu}(x)=\sum_{m=0}^{\infty} \frac{(x / 2)^{\nu+2 m}}{m ! \Gamma(\nu+m+1)}
$$


which is real valued for $\nu$ real on the domain $x>0$ and it is positive for $\nu \geq-1$ on the same domain. Now we observe that

$$
I_{\nu+1}(x)=\sum_{m=0}^{\infty} \frac{(x / 2)^{\nu+2 m+1}}{m ! \Gamma(\nu+m+2)}<\frac{x}{2(\nu+1)} I_{\nu}(x)
$$

this result is an improvement over Nasell [13] for $x<\nu+2$. Now in the series representation of $I_{\nu}(x)$, replacing $\nu$ by $(\nu+1)$, multiplying by $2(\nu+1)$, and subtracting the resulting expression from $x I_{\nu}(x)$, we have

$$
\begin{aligned}
x I_{\nu}(x)-2(\nu+1) I_{\nu+1}(x) & =\sum_{m=0}^{\infty} \frac{(x / 2)^{\nu+2 m+3}}{m ! \Gamma(\nu+m+3)} \\
& <\frac{x^{3}}{4(\nu+1)(\nu+2)} I_{\nu}(x) .
\end{aligned}
$$

Hence

$$
\frac{x}{2(\nu+1)}-\frac{x^{3}}{8(\nu+1)^{2}(\nu+2)}<\frac{I_{\nu+1}(x)}{I_{\nu}(x)}, \quad \nu>-1, x>0,
$$

(3.6), (3.7), the recurrence relation (3.5) and integration over $(x, y)$ then give the inequality

$$
\begin{gathered}
\exp \left\{\frac{x^{2}-y^{2}}{4(\nu+1)}\right\}\left(\frac{x}{y}\right)^{\nu}<\frac{I_{\nu}(x)}{I_{\nu}(y)}<\left(\frac{x}{y}\right)^{\nu} \exp \left\{\frac{x^{2}-y^{2}}{4(\nu+1)}+\frac{y^{4}-x^{4}}{32(\nu+1)^{2}(\nu+2)}\right\} \\
\nu>-1, y>x>0 .
\end{gathered}
$$

4. Inequalities for ${ }_{1} F_{1}(a ; b ; x) /{ }_{1} F_{1}(c ; d ; y)$

Let us consider the integral representation

$$
{ }_{1} F_{1}(a ; c ; z)=\frac{\Gamma(c)}{\Gamma(a) \Gamma(c-a)} \int_{0}^{1} e^{-z t} t^{a-1}(1-t)^{c-a-1} d t, \quad c>a>0,
$$

which with the help of the inequality

$$
1-z<e^{-z}<1-z+\frac{z^{2}}{2}, \quad z>0
$$

and Beta integral representation gives

$$
1-\frac{x a}{c}<{ }_{1} F_{1}(a ; c ;-x)<1-\frac{x a}{c}+\frac{x^{2}(a)_{2}}{2(c)_{2}}, \quad c>a>0 .
$$

Now replacing a by $c-a$ and using the transformation formula

$$
{ }_{1} F_{1}(a ; c ; x)=e^{x}{ }_{1} F_{1}(c-a ; c ;-x)
$$


we have the inequality

$$
e^{x}\left[1-\frac{x(c-a)}{c}\right]<{ }_{1} F_{1}(a ; c ; x)<e^{x}\left[1-\frac{x(c-a)}{c}+\frac{x^{2}(c-a)_{2}}{2(c)_{2}}\right], c>a>0 .
$$

Alternatively, (4.3) can be written as

$$
\begin{aligned}
\frac{1}{e^{y}\left[1-\frac{y(c-a)}{c}+\frac{y^{2}(c-a)_{2}}{2(c)_{2}}\right]}<\frac{1}{{ }_{1} F_{1}(a ; c ; y)}<\frac{1}{e^{y}\left[1-\frac{y(c-a)}{c}\right]} \\
0<y<2, c>a>0 .
\end{aligned}
$$

Now, combining (4.3) and (4.4), we have

under the set of conditions $0<x<2,0<y<2, c>a>0$.

$$
\begin{aligned}
e^{x-y}\left[\frac{1-\frac{x(c-a)}{c}}{1-\frac{y(c-a)}{c}+\frac{y^{2}(c-a)_{2}}{2(c)_{2}}}\right] & <\frac{{ }_{1} F_{1}(a ; c ; x)}{{ }_{1} F_{1}(a ; c ; y)} \\
& <e^{x-y}\left[\frac{1-\frac{x(c-a)}{c}+\frac{x^{2}(c-a)_{2}}{2(c)_{2}}}{1-\frac{y(c-a)}{c}}\right],
\end{aligned}
$$

A comparison shows that (4.5) gives stronger bounds than that of Bordelon [3]. As an example let us consider the set of values $x=.1, y=.8, a=.5, c=.8$, for which (4.5) gives

$$
.6075805<\frac{{ }_{1} F_{1}(a ; c ; x)}{{ }_{1} F_{1}(a ; c ; y)}=.6211304<.6837654
$$

whereas Bordelon's [3] result gives

$$
.496583<\frac{{ }_{1} F_{1}(a ; c ; x)}{{ }_{1} F_{1}(a ; c ; y)}=.6211304<1.0 .
$$

Similarly, we have

$$
\begin{aligned}
{\left[\frac{1-\frac{x(c-a)}{c}}{1-\frac{x(d-a)}{d}+\frac{x^{2}(d-a)_{2}}{2(d)_{2}}}\right] } & <\frac{{ }_{1} F_{1}(a ; c ; x)}{{ }_{1} F_{1}(a ; d ; x)} \\
& <\left[\frac{1-\frac{x(c-a)}{c}+\frac{x^{2}(c-a)_{2}}{2(c)_{2}}}{1-\frac{x(d-a)}{d}}\right],
\end{aligned}
$$

under the set of conditions $0<x<2, c>a>0, d>a>0$.

A comparison with Bordelon's [3] result shows that (4.6) not only gives the two sided inequality but it improves the lower bound of Bordelon [3] as well. As an example let us consider the set of values $x=.5, a=.5, c=.7, d=.8$, we have from (4.6)

$$
1.012743<\frac{{ }_{1} F_{1}(a ; c ; x)}{{ }_{1} F_{1}(a ; d ; x)}=1.044038<1.0859729
$$


whereas Bordelon's [3] result gives

$$
.726542<\frac{{ }_{1} F_{1}(a ; c ; x)}{{ }_{1} F_{1}(a ; d ; x)}=1.044038
$$

Acknowledgement: Our sincere thanks are due to the referee for valuable suggestions. Also the first author expresses his thanks to CSIR for financial support.

\section{References}

[1] E. Amos, "Computation of modified Bessel functions and their ratios," Math. Comp. 28 (1974), 239-251.

[2] R. Askey, "One sided approximation to special functions," SIAM Rev. 18 No. 1 (1976), 121-122.

[3] D. J. Bordelon, "Solution to problem 72-15, Inequalities for special functions," D. K. Ross, SIAM Rev. 15 (1972), 666-668.

[4] A. K. Common, "Uniform inequalities for ultraspherical polynomials and Bessel functions of fractional order," J. Approx. Theory, 49 (1985), 137-145.

[5] A. Erdelyi et. al., Tables of Integral Transforms, Vol. I, Mc-Graw Hill, New York (1953).

[6] C. M. Joshi and S. K. Bissu, "Some inequalities of Bessel and modified Bessel functions," J. Aust. Math. Soc. Series A, 51 (1991), 333-342.

[7] C. M. Joshi and S. K. Bissu, "Inequalities for some special functions-I"(communicated).

[8] A. Laforgia, "Inequalities for Bessel functions," J. Comp. Appl. Math. 15 (1986), 75-81.

[9] A. Laforgia, "Bounds for modified Bessel functions," J. Comp. Appl. Math. 34 (1991), 263-267.

[10] A. Laforgia, "Inequalities for some special functions," Proc. of Sixth Southeastern Approx. Theorists Conference (Ed.) George A. Anastassion, Marcel Dekker Inc., (1992), 385-391.

[11] A. Laforgia and M. L. Mathis, "Bounds for Bessel functions," Rend. Del Cir. Mat. Di Palermo Series II 38 (1989), 319-328.

[12] Y. L. Luke, "Inequalities for generalized hypergeometric functions," J. Approx. Theory 5 (1974), 41-65.

[13] I. Nasell, "Inequalities for modified Bessel functions," Math. Comp. 28 (1974), 253-256.

[14] R. B. Paris, "An inequality for the Bessel functions $J_{\nu}(\nu x)$," SIAM J. Moth. Anal. 9 (1984), 203-205.

[15] D. K. Rosenthal, "The shape and stability of a bubble at the axis of a rotating fluid," J. Fluid. Mech. 12 (1962), 358-366.

[16] D. K. Ross, "The stability of a column of liquid to torsial oscillations," Z. Angew. Math. Phys. 21 (1970). 137-140.

[17] D. K. Ross, "Inequalities for special functions," SIAM Rev. 15 (1973), 668-670.

[18] K. M. Siegel, "An inequality involving Bessel functions of argument nearly equal their order," Proc. Amer. Math. Soc. 4 (1953), 858-859.

[19] G. N. Watson, A treatise on the Theory of Bessel Functions, 2nd èdition, Cambridge University Press, 1944.

Department of Mathematics, Sukhadia University, Udaipur, India. 